\title{
SOME MESOSCOPIC RINGS: EXACT SIMULATIONS AND EXPERIMENT
}

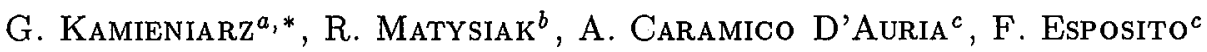 \\ AND C. BENELLI ${ }^{d}$
}

${ }^{a}$ Computational Physics Division, Institute of Physics, A. Mickiewicz University Umultowska 85, 61-614 Poznań, Poland ${ }^{b}$ Institute of Technology, Pedagogical University in Zielona Góra, Poland "Dipartimento di Scienze Fisiche, Università di Napoli "Federico II" Piazzale Tecchio, 80125 Napoli and INFM Unità di Napoli, Italy ${ }^{d}$ Dipartimento di Chimica, Università di Firenze via Maragliano 77, 50144 Firenze, Italy

(Received July 3, 2000; revised version September 25, 2000)

A numerical transfer-matrix approach and an exact diagonalization technique exploiting the point-group symmetry are worked out in the framework of quantum statistical mechanics and group theory for finite rings. They are applied to spin models of the high nuclearity cyclic clusters [Mn(hfac) $\left.{ }_{2} \mathrm{NITPh}\right]_{6}$ and $\mathrm{Ni}_{12}\left(\mathrm{O}_{2} \mathrm{CMe}\right)_{12}(\mathrm{chp})_{12}\left(\mathrm{H}_{2} \mathrm{O}\right)_{6}(\mathrm{THF})_{6}$. The microscopic parameters of both molecules $\left(J / k_{\mathrm{B}}=350 \pm 10 \mathrm{~K}\right.$ and $J / k_{\mathrm{B}}=8.5 \mathrm{~K} \pm 0.5, g=2.23 \pm 0.01$, respectively) are then obtained from a fit of the theoretical susceptibility curves to the experimental results which are supplemented for $\mathrm{Ni}_{12}$ by new low-temperature measurements.

PACS numbers: $75.10 . \mathrm{Jm}, 75.40 . \mathrm{Cx}, 75.40 . \mathrm{Mg}$

\section{Introduction}

The polynuclear clusters make magnetic materials of the size intermediate between that of isolated dimers or trimers and that of bulk magnetic materials [1-5]. These magnetic materials have the mesoscopic scale so that quantum effects may coexist with classical behaviour and new properties can be observed for them. Large metal ion clusters are also present in biological systems (e.g. ferritin [1]) and the modelling of their properties is currently under way. Finally, large assemblies of spins are interesting as real objects to test the theoretical models with finite number of spins.

*corresponding author; e-mail: gjk@phys.amu.edu.pl 
Characterization of the polynuclear magnetic clusters remains a challenging task $[6,7]$. The main difficulty in the theoretical calculations of their magnetic properties is the exponential computational complexity. Quantitative interpretations are available only in a few fortunate cases.

The aim of this paper is to present exact simulation techniques to characterize the compounds consisting of the high nuclearity rings: [ $\left.\mathrm{Mn}(\mathrm{hfac})_{2} \mathrm{NITPh}\right]_{6}$ and $\mathrm{Ni}_{12}\left(\mathrm{O}_{2} \mathrm{CMe}\right)_{12}(\mathrm{chp})_{12}\left(\mathrm{H}_{2} \mathrm{O}\right)_{6}(\mathrm{THF})_{6}$, and to estimate their model parameters in the framework of the Heisenberg spin Hamiltonian. The abbreviations stand for: hfac - hexafluoroacetylacetonate, chp - the anion of 6-chloro-2-pyridone, NITPh - 2-phenyl-4,4,5,5-tetramethyl-4,5-dihydro-1H-imidazolyl-1-oxyl-3-oxide, THF tetrahydrofuran. The complexes are referred to as the supramolecular clusters $\mathrm{Mn}_{6}$ [4] and $\mathrm{N}_{12}$ [2], respectively. Their common feature is the unusual spin $S=12$ ground state, one of the highest spin multiplicity states observed for molecular species $[1,2,4]$.

The $\mathrm{Mn}_{6}$ molecule contains 12 paramagnetic centers $[1,4]$, namely 6 manganese (II) $S=\frac{5}{2}$ ions and 6 organic radicals with non-paired $S=\frac{1}{2}$ electron. The qualitative considerations $[1,4]$ have led to a conclusion that the magnetic properties can be characterized by a strong antiferromagnetic coupling $J>250 \mathrm{~K}$ between the two types of spins.

The $\mathrm{Ni}_{12}$ cluster [2] is a dodecanuclear metallocyclic complex. The compound has two nickel atoms in the asymmetric unit, bound to six oxygen atoms in the distorted octahedral array. The nickel ions are bridged by intersecting $\mathrm{Ni}_{2} \mathrm{O}_{2}$ rings leading to the ferromagnetic coupling estimated previously at $J=13.5 \mathrm{~K}$ [2] and there are no significant intermolecular interactions. The crystal structure of $\mathrm{Ni}_{12}$ resembles that of the decanuclear $\left[\mathrm{Fe}(\mathrm{OMe})_{2}\left(\mathrm{O}_{2} \mathrm{CCH}_{2} \mathrm{Cl}\right)\right]_{10}$, known as the ferric wheel [3], displaying interesting quantum effects in the bulk magnetic measurements.

\section{Theoretical model and simulation techniques}

We consider the rings in the framework of the spin model Hamiltonian

$$
\mathcal{H}=-\sum_{i=1}^{N}\left(J S_{i} \cdot S_{i+1}+g \mu_{\mathrm{B}} B S_{i}^{z}\right),
$$

where $J$ denotes the nearest-neighbour interaction constant (positive for the ferromagnetic coupling), $B$ is the external magnetic field applied along the $z$ direction, $g$ is the corresponding gyromagnetic ratio and $N$ stands for the number of sites in the ring $(N+1 \rightarrow 1)$. The spin value $S_{i}$ may be uniform $\left(S=1\right.$ for $\mathrm{Ni}_{12}$ molecule $)$ or non-uniform (for $\mathrm{Mn}_{6}$ cluster). In the latter case $S=\frac{5}{2}$ for the odd site $i$ and $S=\frac{1}{2}$ for the even site $i$.

First we describe the quantum transfer-matrix (QTM) simulation method for the rings (1), introduced in [8]. Previously QTM was applied to the macroscopic Haldane gap [9] and molecular-based [10] chains with uniform spin $S=1$. It is not subject to any statistical nor systematic errors and the macroscopic limit can be directly evaluated from the largest eigenvalue of the transfer matrix. 
To outline the method for the finite rings, we express Hamiltonian (1) in terms of the spin-pair operators $\mathcal{H}_{i, i+1}$ as

$$
\mathcal{H}=\sum_{i=1}^{N} \mathcal{H}_{i, i+1}=-\sum_{i=1}^{N}\left[J S_{i} \cdot S_{i+1}+\frac{1}{2} g \mu_{\mathrm{B}} B\left(S_{i}^{z}+S_{i+1}^{z}\right)\right] .
$$

If Hamiltonian (2) cannot be directly diagonalized for a given $N$, we may calculate some classical approximants of the thermodynamic functions and then recover the quantum regime, taking the appropriate limit. The thermodynamic functions are related to the free energy

$$
\mathcal{F}=-k_{\mathrm{B}} T \ln \mathcal{Z}=-k_{\mathrm{B}} T \ln \operatorname{Tr} \mathrm{e}^{-\beta \mathcal{H}},
$$

where $\mathcal{Z}$ denotes the canonical partition function. The series of classical approximants of the quantum thermal values can be found, using the general SuzukiTrotter formula [9]. The partition function is calculated from the expression

$$
\mathcal{Z}=\lim _{m \rightarrow \infty} \mathcal{Z}_{m}=\lim _{m \rightarrow \infty} \operatorname{Tr}\left[\prod_{i=1}^{N} \mathrm{e}^{-\beta \mathcal{H}_{i, i+1} / m}\right]^{m},
$$

where the trace is taken over all the configurations of the classical Ising variable $S_{i j}$ (the eigenvalues of $S_{i j}^{z}$ ) on a planar lattice of the size $N \times 2 m$. The periodic boundary conditions $(N+1 \rightarrow 1)$ are imposed to account for the cyclic character of physical objects. The zero-field susceptibility is then evaluated from the second derivative of $(3)$ with respect to field.

The numerical implementation of (4) is based on two global transfer operators $\mathcal{W}_{i}(i=1,2)$ acting in the space $\mathcal{H}^{N}$ which is a direct product of $N$ single-spin spaces $\mathcal{H}_{i}$. The final expression is given by

$$
\mathcal{Z}=\left(\mathcal{W}_{1} \mathcal{W}_{2}\right)^{m}
$$

where

$$
\mathcal{W}_{i}=\left(\mathcal{V}_{i} \mathcal{P}^{+}\right)^{N / 2}, \quad i=1,2
$$

The operators $\mathcal{P}$ and $\mathcal{V}_{i}(i=1,2)$ stand for a unitary shift operator and a local transfer operator, respectively [11]. The explicit form of the operator $\mathcal{V}_{i}$ depends on the choice of the spin operators in Hamiltonian (1) whereas that of $\mathcal{P}$ is universal.

The QTM technique can be applied for model (1) with both the uniform and non-uniform spin variables. However, in the case of the uniform spin variable $S=1$ and $N=12$ (appropriate for $\mathrm{Ni}_{12}$ ), the exact simulations can be accomplished, using simple group-theoretical arguments, an effective coding and numerical diagonalization. The technique is an alternative with respect to that based on the irreducible tensor operator formalism [6] or that exploiting an advanced algebraic and combinatorial approach [12].

The translational symmetry of Hamiltonian (1) can be described in terms of the unitary shift operator $\mathcal{P}$ which commutes with $\mathcal{H}$ and the $z$ component of the total spin $\mathcal{S}^{z}$, and is convenient for the numerical computations. The eigenvalues of $\mathcal{P}$ are the $N$-th order roots of 1 and the corresponding eigenvectors are complex linear combinations of the basis vectors. To avoid complex numbers which increase 
the storage requirements, we have introduced the real operator $\frac{1}{2}\left(\mathcal{P}+\mathcal{P}^{+}\right)$. The invariant subspaces of this operator have bigger dimensions but we can recover the reduction by a factor of $N$, including in our analysis the reflection symmetry. It leaves all previously defined subspaces invariant, and the associated operator $\mathcal{R}$ has the eigenvalues +1 and -1 . In this way the largest invariant subspace has the dimension 6166 for $N=12$ and $S=1$ which is comparable to that found in [12] and much lower than that for the tensor-operator approach [6]. The advantage of our present technique is its effectiveness in calculating the matrix elements. As the task of diagonalization of matrices of this order is not prohibitive for a supercomputer, the whole spectrum of Hamiltonian (1) can be "exactly" calculated. Afterwards any thermodynamic function can be easily obtained using the standard formulae.

\section{Results for the rings $\mathrm{Mn}_{6}$ and $\mathrm{Ni}_{12}$}

The QTM approach is applied here to calculate the susceptibility of the ring with the alternating spins $S_{A}=\frac{5}{2}, S_{B}=\frac{1}{2}$. For a finite $N=12$, the full trace in (4) has to be taken into account and the size of the transfer matrix blows up to 2985984 . We have carefully checked the convergence of our results with respect to length in the Trotter direction and we compared the final estimates with those available in Table 4 of [6]. In order to reach the accuracy up to the 5th decimal place for $2 \leq n=N / 2 \leq 4$ spin pairs, we had to carry out $200 \leq m \leq$ 900 steps in the Trotter direction, depending on $n$ and on temperature. For $n=5$ and the accuracy up to the 4 th decimal place, 50 steps were sufficient. To avoid the excessive amount of the supercomputer time, we have carried out the simulations up to $n=5$ pairs. To characterize $\left[\mathrm{Mn}(\mathrm{hfac})_{2} \mathrm{NITPh}\right]_{6}$ which consists of 6 spin pairs, we have estimated the zero-field susceptibility from the extrapolations of the data for $2 \leq n \leq 5$. A rather good convergence in terms of $n$, sufficient for our purposes, has been achieved, down to $k_{\mathrm{B}} T / J=0.05$. Due to increasing quantum fluctuations, the uncertainty of our prediction at the lowest temperature has raised to the order of $5 \%$. For that reason, to verify our results, we have undertaken the QTM simulations of the system with the full number of $n=6$ spin pairs at $k_{\mathrm{B}} T / J=0.05$. The corresponding numerically "exact" estimate (up to the 4th decimal place) of the zero-field susceptibility is plotted by the full circle in Fig. 1, yielding the excellent confirmation of the approximate calculations $[8,12]$.

In view of the negligible spin-orbit coupling, we have fixed in our fitting procedure $g=2$ and we have determined the best-fit isotropic parameter $J / k_{\mathrm{B}}=$ $350 \pm 10 \mathrm{~K}$. This conclusion is consistent with the existing qualitative estimates $[1,4]$. The temperature dependence of our $\chi T$ predictions for $\left[\mathrm{Mn}(\mathrm{hfac})_{2} \mathrm{NITPh}\right]_{6}$ is displayed in Fig. 1. Numerical data marked by the open circles agree within the error bars with the susceptibility measurements (plotted by dots) of the real compound in question and with the exact value given by the full circle.

The fitting procedure was performed for $\mathrm{Ni}_{12}$, transforming first the experimental data [2] to the usual molar susceptibility units. The latter are plotted for the product $\chi T$ as a function of temperature by the full circles in Fig. 2. These measurements were performed for temperatures higher than $4 \mathrm{~K}$. To extend 


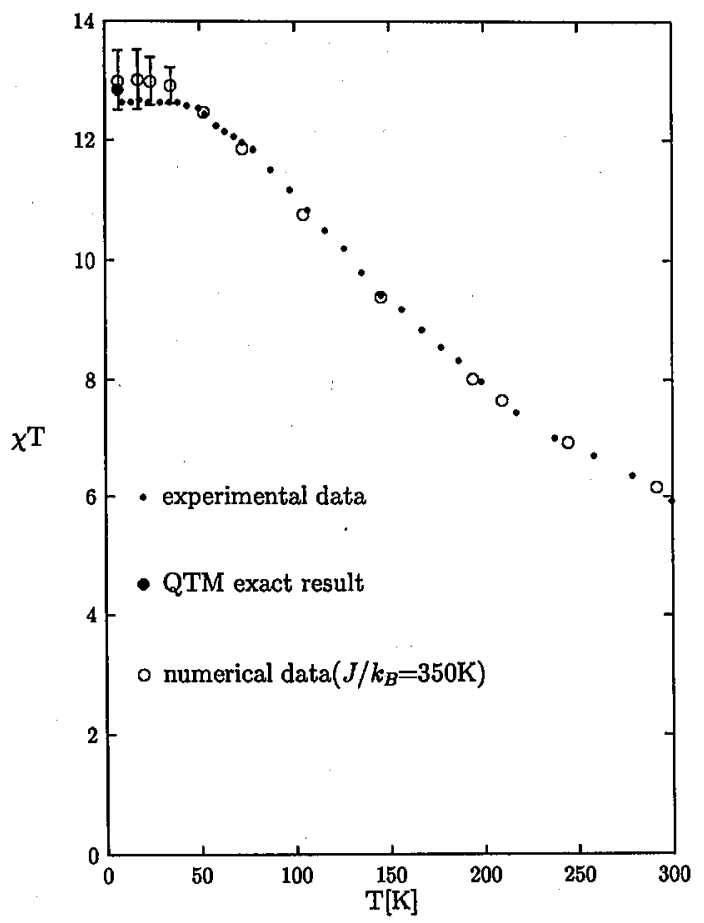

Fig. 1. The temperature dependence of the product $\chi T$ for $\mathrm{Mn}_{6}$ in molar units [emu $\mathrm{K} / \mathrm{mole}$ ] for the monomeric formula. The experimental data are given by the dots and the extrapolated QTM results by the open circles. The full circle shows the numerically exact $\mathrm{QTM}$ estimate at $k_{\mathrm{B}} T / J=0.05$. The error bars are indicated if they exceed the size of the symbols.

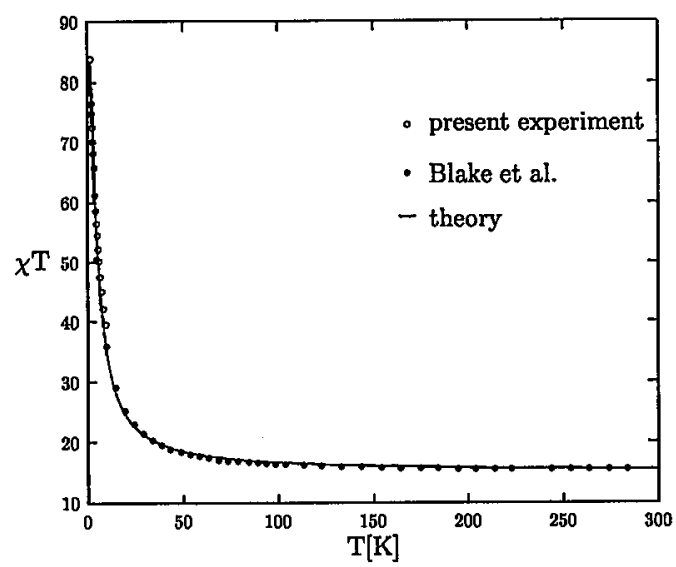

Fig. 2. The temperature behaviour of $\chi T$ for $\mathrm{Ni}_{12}$ in molar units [emu $\mathrm{K} / k_{\mathrm{B}}$ ]. The previous experimental data are plotted by the full circles and the newly reported ones - by the open circles. Our numerical estimates are drawn by the full line. 
the susceptibility behaviour down to lower temperatures, new measurements in the region $1.6-9.5 \mathrm{~K}$ in an effective field of $0.098 \mathrm{~T}$ were made using a Metronique Ingegnerie SQUID with the sample sealed in a teflon holder. Diamagnetic corrections for the sample and the sample holder were applied to the data. The magnetic susceptibility values were corrected to account for the contributions of the non-magnetic atoms. We plot these results for the product $\chi T$ by the open circles in Fig. 2, too. Qualitatively, they agree with those published earlier [2] but they are extended down to $T=1.6 \mathrm{~K}$ with a small temperature decrement. For the data available, the best fit is achieved for the parameters $g=2.23 \pm 0.01$, $J=8.5 \pm 0.5$. The theoretical curve is drawn by the full line and it interpolates the corresponding measured values very well.

\section{Conclusions}

We have worked out the two approaches suitable to characterize the finite temperature magnetic properties of the high nuclearity cyclic spin clusters with large and alternating spins. The QTM technique can provide the numerically exact results if the dimensionality of the multispin space allows the trace operation in the definition of the partition function. It is the case for the $\mathrm{Mn}_{6}$ cluster. If it is not feasible, the technique can be still used after a suitable change of the direction of transfer. We would follow this strategy for $\mathrm{Ni}_{12}$ system but our algebraic group-theoretical approach led us to reduce the dimensionality of the total spin component subspaces to at least 6166 . In this level of complexity the diagonalization task becomes tractable by the present computing platforms. As soon as the eigenstates and eigenvalues are known, all the physical quantities can be calculated exactly at any temperature very quickly. This has led us to characterize quantitatively the $\mathrm{Ni}_{12}$ complex in a very efficient way.

We would like to emphasize that both approaches are effective and can be exploited for other polynuclear clusters.

\section{Acknowledgments}

We would like to thank Prof. Dante Gatteschi, Prof. Roberta Sessoli and Dr. Andrea Caneschi for helpful discussions. This work was supported in part by the Committee for Scientific Research under the grant No. 2 P03B 07514. Numerical calculations were mainly carried out on the platforms of the Supercomputing and Networking Center in Poznan. One of us (G.K.) would like to acknowledge a warm hospitality and the financial support from University of Naples "Federico II" where this work was completed.

\section{References}

[1] D. Gatteschi, A. Caneschi, L. Pardi, R. Sessoli, Science 265, 1054 (1994).

[2] A.J. Blake, C.M. Grant, S. Parsons, J.M. Rawson, R.E.P. Winpenny, J. Chem. Soc., Chem. Commun., 2363 (1994). 
[3] K.L. Taft, C.D. Delfs, G.C. Papaefthymiou, S. Foner, D. Gatteschi, S.J. Lippard, J. Am. Chem. Soc. 116, 823 (1994).

[4] A. Caneschi, D. Gatteschi, J. Laugier, P. Rey, R. Sessoli, C. Zanchini, J. Am. Chem. Soc. 110, 2795 (1988).

[5] A. Müller, S.Q.N. Shah, H. Bögge, M. Schmidtmann, Nature 397, 48 (1999).

[6] D. Gatteschi, L. Pardi, Gazetta Chimica Italiana 123, 231 (1993).

[7] A. Lascialfari, D. Gatteschi, F. Borsa, A. Cornia, Phys. Rev. B 55, 14341 (1997).

[8] G. Kamieniarz, R. Matysiak, A. Caramico D'Auria, F. Esposito, U. Esposito, $J$. Magn. Magn. Mater. 196-197, 915 (1999).

[9] G. Kamieniarz, R. Matysiak, A. Caramico D'Auria, F. Esposito, U. Esposito, Phys. Rev. B 56, 645 (1997).

[10] A. Caramico D'Auria, F. Esposito, U. Esposito, D. Gatteschi, G. Kamieniarz, S. Wałcerz, J. Chem. Phys. 109, 1613 (1998).

[11] T. Delica, H. Leshke, Physica A 168, 736 (1990).

[12] G. Kamieniarz, R. Matysiak, W. Florek, S. Wałcerz, J. Magn. Magn. Mater. 203, 271 (1999). 